\title{
Surface water waves as saddle points of the energy
}

\author{
B. Buffoni* \\ Département de mathématiques \\ Ecole Polytechnique Fédérale, 1015 Lausanne, Suisse \\ Boris.Buffoni@epfl.ch \\ É. Séré \\ CEREMADE, Université Paris-Dauphine \\ Place du Maréchal de Lattre de Tassigny \\ 75775 Paris Cedex 16 \\ Eric.Sere@ceremade.dauphine.fr \\ J. F. Toland $\ddagger$ \\ Department of Mathematical Sciences, University of Bath \\ Claverton Down, Bath, BA2 7AY, UK \\ jft@maths.bath.ac.uk
}

August 2002

\begin{abstract}
By applying the mountain-pass lemma to an energy functional, we establish the existence of two-dimensional water waves on the surface of an infinitely deep ocean in a constant gravity field. The formulation used, which is due to K. I. Babenko [3, 4] (and later to others, independently), has as its independent variable an amplitude function which gives the surface elevation. Its nonlinear term is purely quadratic but it is nonlocal because it involves the Hilbert transform. Moreover the energy functional from which it is derived is rather degenerate and offers an important challenge in the calculus of variations.

In the present treatment the first step is to truncate the integrand, and then to penalize and regularize it. The mountain-pass lemma gives the existence of critical points of the resulting problem. To check that, in the limit of vanishing regularization, the critical points converge to a non-trivial water wave, we need $a$ priori estimates and information on their Morse index in the spirit of the work by Amann and Zehnder [1] (see also [14]). The amplitudes of the waves so obtained
\end{abstract}

*Supported by a grant of the Swiss National Science Foundation.

${ }^{\dagger}$ Membre de l'Institut Universitaire de France.

${ }^{\ddagger}$ Supported by an EPSRC Senior Fellowship. All three authors enjoyed the possibility of collaborating during the "Clay Mathematics Institute Symposium and EuroWorkshop on Hamiltonian Systems" at Edinburgh in May 2001. 
are compared with those obtained from the bifurcation argument of Babenko, and are found to extend the parameter range where existence is known by analytical methods. We also compare our approach with the minimization-under-constraint method used by R. E. L. Turner [25].

2000 MSC: 76B15, 35B38, 58E50.

Keywords: periodic water waves, free-boundary problem, variational method, critical-point theory, saddle point, mountain pass, Morse index.

\section{Introduction}

The water-wave problem is the determination of wave profiles compatible with Bernoulli's theorem which implies that the pressure at the surface of a two-dimensional, infinitely deep, irrotational, incompressible flow under gravity is constant (surface tension is not considered in this paper). The physical parameters are the wave speed, the wavelength, and gravity (acting vertically downwards). After some rescaling, they can be chosen equal to be, respectively, $1,2 \pi$ and $\lambda$. (Note that $\lambda$ is now the only (dimensionless) parameter in the problem.)

Consider such a wave in a reference frame propagating with the speed of the wave. The constant-pressure condition then has the form

$$
\frac{1}{2}|\overrightarrow{\boldsymbol{v}}|^{2}+\lambda y=\frac{1}{2} \text { on the free boundary } y=\eta(x),
$$

where $\eta$ is a smooth $2 \pi$-periodic function and $\overrightarrow{\boldsymbol{v}}$ is a smooth two-dimensional velocity field that is divergence free and irrotational on the domain $\{(x, y): y \leq \eta(x)\}$. The asymptotic velocity at infinite depth is

$$
\lim _{y \rightarrow-\infty} \overrightarrow{\boldsymbol{v}}(x, y)=(-1,0) \text { uniformly in } x \in \mathbb{R} .
$$

If such a wave exists then the energy, kinetic plus potential, in one wavelength is

$$
E=\int_{x=-\pi}^{\pi} \int_{y=-\infty}^{\eta(x)} \frac{1}{2}\left\{|\overrightarrow{\boldsymbol{v}}|^{2}-1\right\} d x d y+\int_{-\pi}^{\pi} \frac{1}{2} \lambda \eta^{2}(x) d x,
$$

where 1 is subtracted from $|\overrightarrow{\boldsymbol{v}}|^{2}$ so that the integral is finite (this is related to the choice of the constant $1 / 2$ on the right-hand side of (1)).

P. R. Garabedian [15] argued that variational methods applied to $E$ should yield the existence of water waves. However Turner [25] pointed out some technical difficulties with the idea and introduced a different variational approach which led to the existence of periodic and solitary waves on the surface of a fluid layer of finite depth. (In the absence of surface tension, it is known that solitary waves do not exist if the depth is infinite [13]).

More recently Babenko [3, 4] introduced a new variational formulation of the periodic water-wave problem, but used non-variational methods to study it. (See also [18].) Plotnikov [19] discovered the analogous formulation for solitary waves. (See [23] for references to other independent discoveries of Babenko's formulation.) The advantage of this formulation is that it involves a quadratic equation for a function of a single real variable; the difficulty is that it involves the Hilbert transform which is a non-local operator. 
Underlying our approach is the idea of adapting Turner's ideas and the mountain-pass lemma to the Babenko formulation. Like Turner, we get the existence of small-amplitude water waves. However our method works globally if, roughly speaking, the gravitational force field $\lambda$ in the Bernoulli boundary condition vanishes a little below the maximal height of a Stokes' wave. Only when precise estimates are needed to deal with constant gravity fields is the argument forced to become local. A bifurcation-theoretic variational approach to the Babenko formulation was given in [8] where a Lyapunov-Schmidt reduction, followed by a study of the reduced problem in terms of finite-dimensional constrained optimization, was used. However this is a strictly local method which avoids the essential difficulties of the infinite-dimensional formulation and it gives no quantitative information about the solutions found.

In the end we prove the existence of a nonzero symmetric wave for some $\lambda_{*} \leq 0.99$. This result is not a consequence of the local existence theorem obtained by Babenko [3]. Indeed, in Babenko's paper, solutions are found for $\lambda_{*}=1 /\left(1+\varepsilon^{2}\right)$ with $\varepsilon \in(0,1 / 25]$, i.e. $\lambda_{*} \in[0.9984,1)$. Note that $\lambda_{*}=0.99$ corresponds to $2 \epsilon=0.2$, which, for a small wave, is a good approximation of the trough-to-crest height (for the extreme wave, this height is approximatively $0.8868[7,18]$ ). Much more is known from global continuation methods $[7,8,9]$, from numerical investigation $[12,18]$ and from computer assisted proofs $[5,6]$. However the present work may be a small step towards a better understanding of large-amplitude water waves from a variational viewpoint.

Note added in proof. Since this paper was completed we have developed a general theory of quasi-linear problems in an abstract Hilbert-space setting (see [10]) which is sufficiently general to cover, for example, the the existence question for periodic capillarygravity waves with its awkard curvature term that represents surface-tension effects. In the abstract version of the theory, we stay closer to the spirit of Turner's work and seek minimizers, rather than mountain pass solutions.

\section{Preliminaries}

Let $L_{2 \pi}^{p}, 1 \leq p<\infty$, denote the linear space of $2 \pi$-periodic, real-valued, measurable 'functions' on $\mathbb{R}$ with

$$
\|u\|_{L_{2 \pi}^{p}}=\left\{\int_{-\pi}^{\pi}|u|^{p} d x\right\}^{1 / p}<\infty \text { if } p<\infty,
$$

and essentially bounded if $p=\infty$. The space of functions $u \in L_{2 \pi}^{2}$ with $u^{\prime}$ also in $L_{2 \pi}^{2}$ is denoted by $W_{2 \pi}^{1,2}$. Denote by $C_{2 \pi}^{\infty}$ the space of $2 \pi$-periodic functions $u$ which are infinitely differentiable.

Let the Fourier coefficients of $u \in L_{2 \pi}^{1}$ with respect to the orthonormal basis $\left\{(2 \pi)^{-\frac{1}{2}} e^{i k t}\right.$ : $k \in \mathbb{Z}\}$ be denoted by $\hat{u}_{k}, k \in \mathbb{Z}$. Then $\hat{u}_{-n}=\overline{\hat{u}}_{n}$, since $u$ is real, and $L_{2 \pi}^{2}$ is a real Hilbert space with inner product

$$
\langle u, v\rangle=\sum_{n \in \mathbb{Z}} \hat{u}_{n} \overline{\hat{v}_{n}}
$$

For $u \in L_{2 \pi}^{1}$ let

$$
[u]=\frac{1}{2 \pi} \int_{-\pi}^{\pi} u(t) d t=\frac{1}{\sqrt{2 \pi}} \hat{u}_{0}
$$


In this notation the fractional order Sobolev space $H^{\frac{1}{2}}$ is the Hilbert space of functions $u \in L_{2 \pi}^{1}$ with norm given by

$$
\|u\|_{H^{\frac{1}{2}}}^{2}=\hat{u}_{0}^{2}+\sum_{k \in \mathbb{Z}}\left|k \| \hat{u}_{k}\right|^{2}<\infty .
$$

The periodic Hilbert transform of an $L_{2 \pi}^{1}$-function $u$ is defined for almost all $x \in \mathbb{R}$ by the Cauchy principle value integral

$$
\mathcal{C} u(x)=\frac{1}{2 \pi} \int_{-\pi}^{\pi} u(y) \cot \left(\frac{1}{2}(x-y)\right) d y .
$$

It is well known [26] that $\mathcal{C}: L_{2 \pi}^{p} \rightarrow L_{2 \pi}^{p}$ is a bounded linear operator if $1<p<\infty, \mathcal{C}$ does not map $L_{2 \pi}^{p}$ into itself when $p=1$ and $\infty$, and the Hilbert transform of an infinitely differentiable function is infinitely differentiable. Moreover $\mathcal{C}$ is a Fourier multiplication operator on $L_{2 \pi}^{p}, p>1$, in the sense that

$$
(\widehat{\mathcal{C u}})_{0}=0 \text { and }(\widehat{\mathcal{C u}})_{k}=-i \operatorname{sgn}(k) \hat{u}_{k}, \quad k \in \mathbb{Z} \backslash\{0\}, \text { when } u \in L_{2 \pi}^{p}, p>1 ;
$$

equivalently,

$$
\mathcal{C}(\cos n t)=\sin n t \text { for } n \geq 0 \text { and } \mathcal{C}(\sin n t)=-\cos n t \text { for } n \geq 1 \text {. }
$$

From this it is clear that $u \mapsto \mathcal{C} u^{\prime}$ is symmetric in the sense that

$$
\left\langle u, \mathcal{C} v^{\prime}\right\rangle=\left\langle\mathcal{C} u^{\prime}, v\right\rangle \text { for all } u, v \in C_{2 \pi}^{\infty} \text {. }
$$

The Hilbert transform has a geometric interpretation in the complex plane. Suppose that $w \in W_{2 \pi}^{1,2}$ and that

$$
w(t)=\sum_{n=0}^{\infty}\left(a_{n} \cos n t+b_{n} \sin n t\right), \quad a_{n}, b_{n} \in \mathbb{R}, \quad b_{0}=0 .
$$

Then the complex-valued function

$$
\begin{aligned}
i a_{0} & +\sum_{n=1}^{\infty} i\left(a_{n}+i b_{n}\right) e^{-i n(\phi+i \psi)} \\
& =\sum_{n=1}^{\infty}\left(a_{n} \sin n \phi-b_{n} \cos n \phi\right) e^{n \psi}+i \sum_{n=0}^{\infty}\left(a_{n} \cos n \phi+b_{n} \sin n \phi\right) e^{n \psi}
\end{aligned}
$$

is holomorphic on the open half plane $\{\phi+i \psi \in \mathbb{C}: \psi<0\}$, its trace on the boundary $\{\phi+i 0: \phi \in \mathbb{R}\}$ is $\mathcal{C} w(\phi)+i w(\phi)$ and it converges to $i a_{0}$ as $\psi \rightarrow-\infty$. Since $\left\{a_{n}\right\}$ and $\left\{b_{n}\right\}$ are square-summable, it is well known [26] that $\mathcal{C} w(\phi)+i w(\phi)=0$ on a set of positive measure if and only if $\left\{a_{n}\right\}$ and $\left\{b_{n}\right\}$ are both zero sequences.

Finally we mention an alternative way of interpreting the Hilbert transform in the framework of complex analysis. The function $w$, given by (6), and $\mathcal{C} w$ can be extended to the open unit disc $\mathcal{D}=\{z \in \mathbb{C}:|z|<1\}$ by writing $z=r e^{i t}$ with $0 \leq r<1$ and $t \in \mathbb{R}$ in such a way that $w(t)+i \mathcal{C} w(t)$ is the trace on the unit circle $(r=1)$ of the holomorphic function

$$
a_{0}+\sum_{n=1}^{\infty}\left(a_{n}-i b_{n}\right) z^{n}, \quad z \in \mathcal{D} .
$$

Observe that the value of this extension at $z=0$ is $a_{0}=[w]$ and the trace on the circle is non-zero almost everywhere if $w \not \equiv 0$. 


\section{Variational Formulation}

Our approach is based on the following form of the water-wave problem: to find $(\lambda, w)$ such that

$$
\left\{\begin{array}{l}
\frac{1}{2}\left\{w^{\prime 2}+\left(1+\mathcal{C} w^{\prime}\right)^{2}\right\}^{-1}+\lambda w=\frac{1}{2} \\
1+\mathcal{C} w^{\prime}>0, \quad w \in C_{2 \pi}^{\infty}, \quad \lambda>0
\end{array}\right.
$$

Given a solution $w$ of (7) in the form (6), we obtain a solution of (1) heuristically as follows. For $\phi \in \mathbb{R}$ and $\psi \leq 0$, define

$$
\begin{aligned}
& \widetilde{x}(\phi, \psi)=\phi+\sum_{n=1}^{\infty}\left(a_{n} \sin n \phi-b_{n} \cos n \phi\right) e^{n \psi}, \\
& \widetilde{y}(\phi, \psi)=\psi+\sum_{n=0}^{\infty}\left(a_{n} \cos n \phi+b_{n} \sin n \phi\right) e^{n \psi}
\end{aligned}
$$

The function $\widetilde{y}$ is harmonic and is an extension of $w$ in the sense that $w(t) \equiv \widetilde{y}(t, 0)$; moreover $\widetilde{y}(\phi, \psi)-\psi$ is uniformly bounded on $\{(\phi, \psi): \phi \in \mathbb{R}, \psi \leq 0\}$. In the same way, $\widetilde{x}$ is a harmonic extension of $t+\mathcal{C} w(t)$, so that $t+\mathcal{C} w(t) \equiv \widetilde{x}(t, 0)$ and $\widetilde{x}(\phi, \psi)-\phi$ is uniformly bounded. Moreover the map $\phi+i \psi \rightarrow \widetilde{x}+i \widetilde{y}$ is holomorphic on $\{\phi+i \psi \in \mathbb{C}: \psi<0\}$. Equivalently, $\widetilde{x}$ and $\widetilde{y}$ are harmonic conjugates and satisfy the Cauchy-Riemann equations:

$$
\partial_{\phi} \widetilde{x}=\partial_{\psi} \widetilde{y} \text { and } \partial_{\psi} \widetilde{x}=-\partial_{\phi} \widetilde{y} \text { for all } \phi \in \mathbb{R}, \psi \leq 0 \text {. }
$$

New variables $(x, y)$ can now be defined by writing

$$
(x, y)=(\widetilde{x}(\phi, \psi), \widetilde{y}(\phi, \psi)) .
$$

We find that the half-plane $\{(\phi, \psi): \phi \in \mathbb{R}, \psi \leq 0\}$ is sent injectively onto $\{(x, y): x \in$ $\mathbb{R}, y \leq \eta(x)\}$, where $\eta$ is given implicitly by

$$
\eta(t+\mathcal{C} w(t))=w(t)
$$

and the velocity field is defined by

$$
\overrightarrow{\boldsymbol{v}}=\left(\partial_{x} \phi, \partial_{y} \phi\right)=\left(\partial_{y} \psi,-\partial_{x} \psi\right)=\left\{\left(\partial_{\phi} \widetilde{x}\right)^{2}+\left(\partial_{\phi} \widetilde{y}\right)^{2}\right\}^{-1}\left(\partial_{\phi} \widetilde{x}, \partial_{\phi} \widetilde{y}\right)
$$

(since $\phi+i \psi \rightarrow \widetilde{x}+i \widetilde{y}$ is holomorphic). Hence, as functions of $x$ and $y, \phi$ can be regarded as a velocity potential and $\psi$ as a stream function. Clearly, when $\psi=0$ and $\phi=t \in \mathbb{R}$,

$$
|\overrightarrow{\boldsymbol{v}}|^{2}=\left\{\left(\partial_{\phi} \widetilde{x}\right)^{2}+\left(\partial_{\phi} \widetilde{y}\right)^{2}\right\}^{-1}=\left\{\left(1+\mathcal{C} w^{\prime}(t)\right)^{2}+w^{\prime}(t)^{2}\right\}^{-1}
$$

and $y=\widetilde{y}(t, 0)=w(t)=\eta(x)$. This intuitively explains the equivalence between (1) and (7).

Note that (7) is not a variational equation as it stands. However Babenko [4] found that the first equation of $(7)$ is satisfied by any $w \in C_{2 \pi}^{\infty}$ that satisfies

$$
\mathcal{C} w^{\prime}=\lambda\left\{w+w \mathcal{C} w^{\prime}+\mathcal{C}\left(w w^{\prime}\right)\right\}
$$


which is the Euler equation of the functional

$$
J(w)=\int_{-\pi}^{\pi} w \mathcal{C} w^{\prime}-\lambda w^{2}\left(1+\mathcal{C} w^{\prime}\right) d t
$$

Recently Toland [23] (improving on [21]) observed that all smooth, non-constant solutions of (8) automatically satisfy the remaining condition in (7). The energy (2) of the corresponding wave, when written in terms of $w$ and $\lambda$, is $-2 J(w)$. (The sign in front of $J$ is chosen to ensure positive definiteness in Morse index and other calculations later.)

The simple form of $J$, by comparison with (2), leads one naturally to enquire if water waves can be found using the direct method of the calculus of variations on (9). The present work shows that this is indeed possible. The waves which emerge are small, but not too small. In the next three sections we study a functional more general than $J$, and in the final section we specialize to $J$.

\section{Truncation, Penalization and Regularization}

Suppose that $f \in C^{\infty}(\mathbb{R}), R \in(0, \infty]$ and that $\rho:\left[0, \pi R^{2}\right) \rightarrow \mathbb{R}$ is $C^{\infty}$, convex with $\rho(0)=\rho^{\prime}(0)=0$ and $\rho(s) \rightarrow \infty$ as $s \nearrow \pi R^{2}$. Consider the real-valued functional

$$
\begin{aligned}
& \mathcal{J}(w)=-\int_{-\pi}^{\pi}\left\{f(w)\left(1+\mathcal{C} w^{\prime}\right)+w\right\} d t+\rho\left(\int_{-\pi}^{\pi} w \mathcal{C} w^{\prime} d t\right) \\
& \text { for } \quad w \in W_{2 \pi}^{1,2} \text { and } \int_{-\pi}^{\pi} w \mathcal{C} w^{\prime} d t<\pi R^{2} .
\end{aligned}
$$

In the case when $R=\infty, \rho \equiv 0$ and $f(w)=\lambda w^{2}-w$, the functional $\mathcal{J}$ coincides with $J$.

The idea is to replace the function $\lambda w^{2}-w$ in $J$ with its truncation $f$ in $\mathcal{J}$ so that $\inf \left(-f^{\prime}\right)>0$. This property will be very important when getting a priori estimates and when studying the regularity of the critical points. Note that, as observed in [11], much of the structure of the problem persists for general $f$. The role of the penalization term $\rho\left(\int_{-\pi}^{\pi} w \mathcal{C} w^{\prime} d t\right)$ comes in proving the estimates needed to show that when $w$ is a mountainpass critical point of the regularized $\mathcal{J}_{\epsilon}$ (see $(20)$ ), the function $\lambda w^{2}-w$ is unaffected by the truncation. In fact it allows us to work in the domain $\left\{w \in W_{2 \pi}^{1,2}: \int_{-\pi}^{\pi} w \mathcal{C} w^{\prime} d t<\pi R^{2}\right\}$ by preventing a mountain-pass critical point from approaching the boundary. Alternatively, we could have used the Hampwile theorem [20] in $\left\{w \in W_{2 \pi}^{1,2}: \int_{-\pi}^{\pi} w \mathcal{C} w^{\prime} d t<\pi R^{2}\right\}$.

Critical points $w \in W_{2 \pi}^{1,2}$ of $\mathcal{J}$ with $\int_{-\pi}^{\pi} w \mathcal{C} w^{\prime} d t<\pi R^{2}$ satisfy $\mathcal{J}^{\prime}(w) \phi=0$ for all $\phi \in W_{2 \pi}^{1,2}$, where

$$
\mathcal{J}^{\prime}(w) \phi=-\int_{-\pi}^{\pi} \phi\left\{1+f^{\prime}(w)\left(1+\mathcal{C} w^{\prime}\right)+\mathcal{C}(f(w))^{\prime}\right\} d t+2 \rho^{\prime}\left(\int_{-\pi}^{\pi} w \mathcal{C} w^{\prime} d t\right) \int_{-\pi}^{\pi} \phi \mathcal{C} w^{\prime} d t
$$

It follows that critical points satisfy

$$
\begin{aligned}
& -\left\{f^{\prime}(w)\left(1+\mathcal{C} w^{\prime}\right)+\mathcal{C}\left(f^{\prime}(w) w^{\prime}\right)+1\right\}+2 \rho^{\prime}\left(\int_{-\pi}^{\pi} w \mathcal{C} w^{\prime} d t\right) \mathcal{C} w^{\prime}=0 \\
& w \in W_{2 \pi}^{1,2}, \quad \int_{-\pi}^{\pi} w \mathcal{C} w^{\prime} d t<\pi R^{2}
\end{aligned}
$$


For $\phi \in W_{2 \pi}^{1,2}$, the quadratic form $\mathcal{J}^{\prime \prime}(w)(\phi, \phi)$ is given by

$$
\begin{aligned}
\mathcal{J}^{\prime \prime}(w)(\phi, \phi) & =-\int_{-\pi}^{\pi} \phi\left\{f^{\prime \prime}(w)\left(1+\mathcal{C} w^{\prime}\right) \phi+f^{\prime}(w) \mathcal{C} \phi^{\prime}+\mathcal{C}\left(f^{\prime}(w) \phi\right)^{\prime}\right\} d t \\
& +2 \rho^{\prime}\left(\int_{-\pi}^{\pi} w \mathcal{C} w^{\prime} d t\right) \int_{-\pi}^{\pi} \phi \mathcal{C} \phi^{\prime} d t+4 \rho^{\prime \prime}\left(\int_{-\pi}^{\pi} w \mathcal{C} w^{\prime} d t\right)\left(\int_{-\pi}^{\pi} \phi \mathcal{C} w^{\prime} d t\right)^{2} .
\end{aligned}
$$

In the light of relation, $E=-2 J$, between (2) and (9), smooth solutions of (11) should correspond to water waves when $\rho \equiv 0$ and $f(w)=\lambda w^{2}-w$. The proof of the general result, which is new even for the special case, has an immediate generalization to the regularized functional introduced in (20).

Proposition 1. If $w \in C_{2 \pi}^{\infty}$ is a solution of (11) then

$$
f^{\prime}(w)\left(w^{\prime 2}+\left(1+\mathcal{C} w^{\prime}\right)^{2}\right)+1-\rho^{\prime}\left(\int_{-\pi}^{\pi} w \mathcal{C} w^{\prime} d t\right)\left(w^{\prime 2}+\left(1+\mathcal{C} w^{\prime}\right)^{2}-1\right) \equiv 0 .
$$

In particular, if $\rho \equiv 0$, then $f^{\prime}(w(t))<0$ and $\left\{w^{\prime 2}+\left(1+\mathcal{C} w^{\prime}\right)^{2}\right\}^{-1} \in L_{2 \pi}^{\infty}$.

Proof. Let $w \in C_{2 \pi}^{\infty}$ and let

$$
W^{*}=i\left(1+\mathcal{C} w^{\prime}\right)+w^{\prime} .
$$

Then as we saw in Section $2, W^{*}$ is the trace on the circle of a function $W$ holomorphic on the unit disc and $\left|W^{*}\right|$ is non-zero almost everywhere on $[-\pi, \pi]$. Now suppose that $v \in C_{2 \pi}^{\infty}$, so that $v+i \mathcal{C} v$ is the trace on the boundary of some function $V$ holomorphic on the disc with $V(0)=[v]$. Let $\Phi=i W V$ and note from (14) that

$$
W(0)=i \text {. }
$$

Then $\Phi(0)=-[v] \in \mathbb{R}$ and its trace satisfies $\Phi^{*}=\phi+i \mathcal{C} \phi$ for some $\phi \in C_{2 \pi}^{\infty}$ with $[\phi]=[-v]$. Hence

$$
i W^{*}(v+i \mathcal{C} v)=\phi+i \mathcal{C} \phi \text { and } v=\Im\left(\frac{\phi+i \mathcal{C} \phi}{W^{*}}\right) .
$$

We conclude that, for any $w \in C_{2 \pi}^{\infty}$ the range of the mapping

$$
\phi \mapsto \Im\left(\frac{\phi+i \mathcal{C} \phi}{W^{*}}\right), \quad \phi \in C_{2 \pi}^{\infty},
$$

includes all $v \in C_{2 \pi}^{\infty}$ and, for such $v,[v]=-[\phi]$.

Let $\Re z$ and $\Im z$ denote the real and imaginary parts of $z \in \mathbb{C}$ and suppose that $w \in C_{2 \pi}^{\infty}$ satisfies (11). If $\phi \in C_{2 \pi}^{\infty}$ then clearly

$$
\begin{aligned}
\mathcal{J}^{\prime}(w) \phi= & \int_{-\pi}^{\pi}\left(f^{\prime}(w)\left\{w^{\prime} \mathcal{C} \phi-\phi\left(1+\mathcal{C} w^{\prime}\right)\right\}-\phi\right) d t \\
& -\rho^{\prime}\left(\int_{-\pi}^{\pi} w \mathcal{C} w^{\prime} d t\right) \int_{-\pi}^{\pi}\left\{w^{\prime} \mathcal{C} \phi-\phi\left(1+\mathcal{C} w^{\prime}\right)+\phi\right\} d t \\
= & \int_{-\pi}^{\pi}\left\{f^{\prime}(w)\left|W^{*}\right|^{2} \Im\left(\frac{\phi+i \mathcal{C} \phi}{W^{*}}\right)-\phi\right\} d t \\
& -\rho^{\prime}\left(\int_{-\pi}^{\pi} w \mathcal{C} w^{\prime} d t\right) \int_{-\pi}^{\pi}\left\{\left|W^{*}\right|^{2} \Im\left(\frac{\phi+i \mathcal{C} \phi}{W^{*}}\right)+\phi\right\} d t .
\end{aligned}
$$


Therefore for all $\phi \in C_{2 \pi}^{\infty}$

$$
\begin{aligned}
\mathcal{J}^{\prime}(w) \phi=\int_{-\pi}^{\pi}\left(\left|W^{*}\right|^{2} f^{\prime}(w)\right. & +1) \Im\left(\frac{\phi+i \mathcal{C} \phi}{W^{*}}\right) d t \\
& -\rho^{\prime}\left(\int_{-\pi}^{\pi} w \mathcal{C} w^{\prime} d t\right) \int_{-\pi}^{\pi}\left(\left|W^{*}\right|^{2}-1\right) \Im\left(\frac{\phi+i \mathcal{C} \phi}{W^{*}}\right) d t=0 .
\end{aligned}
$$

Since $\phi$ is arbitrary, the first assertion follows. Now suppose that $\rho \equiv 0$. Then

$$
w^{\prime 2}+\left(1+\mathcal{C} w^{\prime}\right)^{2} \equiv\left|f^{\prime}(w)\right|^{-1} .
$$

Since $f, w$ and $\mathcal{C} w$ are smooth, it follows that $f^{\prime}(w)$ is nowhere zero and the result follows.

When $f(w)=\lambda w^{2}-w$, equation (13) can be written in the form

$$
\frac{1}{2\left(w^{\prime 2}+\left(1+\mathcal{C} w^{\prime}\right)^{2}\right)}+\frac{\lambda}{1+\rho^{\prime}\left(\int_{-\pi}^{\pi} w \mathcal{C} w^{\prime} d t\right)} w=\frac{1}{2} .
$$

When (17) is compared with (7) we see that the parameter $\lambda$ has been replaced by the $a$ priori unknown value

$$
\frac{\lambda}{1+\rho^{\prime}\left(\int_{-\pi}^{\pi} w \mathcal{C} w^{\prime} d t\right)} \leq \lambda
$$

However the introduction of $\rho$ will in the end yield an a priori bound on the norm of $w$. In other words, $\rho$ plays the same role as a constraint in the calculus of variations.

For general $f,(13)$ still has a geometric interpretation, because it can be written formally as

$$
\frac{1}{2\left(w^{\prime 2}+\left(1+\mathcal{C} w^{\prime}\right)^{2}\right)}+\frac{f^{\prime}(w)}{2\left\{1+\rho^{\prime}\left(\int_{-\pi}^{\pi} w \mathcal{C} w^{\prime} d t\right)\right\}}=\frac{\rho^{\prime}\left(\int_{-\pi}^{\pi} w \mathcal{C} w^{\prime} d t\right)}{2\left\{1+\rho^{\prime}\left(\int_{-\pi}^{\pi} w \mathcal{C} w^{\prime} d t\right)\right\}}
$$

Thus a Bernoulli condition still holds but the gravitational force field has now been replaced by a potential field of force pointing vertically downwards with intensity

$$
\frac{f^{\prime \prime}(y)}{2\left\{1+\rho^{\prime}\left(\int_{-\pi}^{\pi} w \mathcal{C} w^{\prime} d t\right)\right\}} .
$$

As before, $y$ is the vertical coordinate (the height) of the surface above some horizontal axis.

Observe that, when $\lambda=0$ and therefore $f(w)=\lambda w^{2}-w=-w$, every constant $w$ is a solution of (11). This family of "trivial" solutions makes the problem unnecessarily complicated. To eliminate these solutions and to remove the translation invariance of the problem, we work in the subspace $H$ of $W_{2 \pi}^{1,2}$ consisting of even functions of zero mean with norm given by

$$
\|w\|_{H}^{2}=\int_{-\pi}^{\pi}\left|w^{\prime}(t)\right|^{2} d t
$$

A critical point $w$ of $\mathcal{J}$ restricted to $H$ satisfies almost everywhere

$$
f^{\prime}(w)\left(1+\mathcal{C} w^{\prime}\right)+\mathcal{C}\left(f^{\prime}(w) w^{\prime}\right)+1-2 \rho^{\prime}\left(\int_{-\pi}^{\pi} w \mathcal{C} w^{\prime} d t\right) \mathcal{C} w^{\prime}=c, \quad \int_{-\pi}^{\pi} w \mathcal{C} w^{\prime} d t<\pi R^{2}
$$

for some constant $c$. It can also give rise to a water wave, as the next result shows. 
Proposition 2. Let $w$ be a smooth critical point of $\mathcal{J}$ restricted to $H$. Then for the constant $c$ in (18),

$$
f^{\prime}(w)\left\{w^{\prime 2}+\left(1+\mathcal{C} w^{\prime}\right)^{2}\right\}+1-\rho^{\prime}\left(\int_{-\pi}^{\pi} w \mathcal{C} w^{\prime} d t\right)\left(w^{\prime 2}+\left(1+\mathcal{C} w^{\prime}\right)^{2}-1\right)=c .
$$

Proof. The proof is the same as that of Proposition 1.

When $f(w)=\lambda w^{2}-w,(19)$ can be written

$$
\frac{1}{2\left(w^{2}+\left(1+\mathcal{C} w^{\prime}\right)^{2}\right)}+\frac{\lambda}{1+\rho^{\prime}\left(\int_{-\pi}^{\pi} w \mathcal{C} w^{\prime} d t\right)-c}\left(w-\frac{c}{2 \lambda}\right)=\frac{1}{2}
$$

and the value of $c$ can be found by integrating (18):

$$
c=\frac{\lambda}{\pi} \int_{-\pi}^{\pi} w \mathcal{C} w^{\prime} d t \geq 0 .
$$

This leads us to impose a new condition on $\rho$ :

$$
\rho^{\prime}(s) \geq(\lambda / \pi) s .
$$

If $\rho$ has this property, then $1+\rho^{\prime}\left(\int_{-\pi}^{\pi} w \mathcal{C} w^{\prime} d t\right)-c \geq 1$, and so the coefficient

$$
\tilde{\lambda}=\frac{\lambda}{1+\rho^{\prime}\left(\int_{-\pi}^{\pi} w \mathcal{C} w^{\prime} d t\right)-c}
$$

is less than $\lambda$. The function $\tilde{w}:=w-c /(2 \lambda)$ is then a solution of the Bernoulli equation (7) in which $\lambda$ is replaced by $\tilde{\lambda}$.

Now, in addition to restricting $\mathcal{J}$ defined by (10) to $H$, we add a regularizing term. Consider the regularized functional $\mathcal{J}_{\epsilon}$ defined by

$$
\mathcal{J}_{\epsilon}(w)=\epsilon I(w)+\mathcal{J}(w), \quad w \in H
$$

where

$$
I(w)=\int_{-\pi}^{\pi}\left|w^{\prime}\right|^{2} d t, \quad w \in H .
$$

Then critical points $w \in H$ of $\mathcal{J}_{\epsilon}$ such that $\int_{-\pi}^{\pi} w \mathcal{C} w^{\prime} d t<\pi R^{2}$ have $w^{\prime} \in W_{2 \pi}^{1,2}$ and satisfy

$$
2 \epsilon w^{\prime \prime}+f^{\prime}(w)\left(1+\mathcal{C} w^{\prime}\right)+\mathcal{C}\left(f^{\prime}(w) w^{\prime}\right)+1-2 \rho^{\prime}\left(\int_{-\pi}^{\pi} w \mathcal{C} w^{\prime} d t\right) \mathcal{C} w^{\prime}=c
$$

almost everywhere for some constant $c$. We will see that that $w=0$ is a non-degenerate minimizer of $\mathcal{J}_{\epsilon}$ when $\epsilon>0$. When $\epsilon=0$, the linear operator $\mathcal{J}_{\epsilon}^{\prime \prime}(0)=\mathcal{J}^{\prime \prime}(0)$ defined on $H$ is not invertible and present knowledge of the regularity of the critical points of $\mathcal{J}$ in larger (i.e. weaker) spaces than $H$ is rather limited [24]. On the other hand, a simple bootstrap argument shows that a solution $w \in W_{2 \pi}^{1,2}$ of $(21)$ has $w \in C_{2 \pi}^{\infty}$. 
In what follows, we do not need the generalization of the pointwise identity (19) to the case $\epsilon>0$; an integral identity that is much easier to prove, namely

$$
\begin{aligned}
0=\mathcal{J}_{\epsilon}^{\prime}(w) \mathcal{C} w^{\prime}=2 \epsilon \int_{-\pi}^{\pi} w^{\prime} \mathcal{C} w^{\prime \prime} d t & -\int_{-\pi}^{\pi} f^{\prime}(w)\left\{\left(\mathcal{C} w^{\prime}\right)^{2}+w^{\prime 2}\right\} d t \\
& -\int_{-\pi}^{\pi} f^{\prime}(w) \mathcal{C} w^{\prime} d t+2 \rho^{\prime}\left(\int_{-\pi}^{\pi} w \mathcal{C} w^{\prime} d t\right) \int_{-\pi}^{\pi} w^{\prime 2} d t
\end{aligned}
$$

suffices. (This integral identity also holds for $\epsilon=0$ and $w \in H$, as it is seen by multiplying (18) by $\mathcal{C} w^{\prime}$ and integrating.) However, for completeness, we show that if $w \in H$ satisfies $\int_{-\pi}^{\pi} w \mathcal{C} w^{\prime} d t<\pi R^{2}$ and is a (necessarily smooth) solution of (21) with $\epsilon>0$, then

$$
\begin{aligned}
2 \epsilon w^{\prime \prime}+ & 2 \epsilon\left\{w^{\prime \prime} \mathcal{C} w^{\prime}-\mathcal{C}\left(w^{\prime} w^{\prime \prime}\right)\right\} \\
& +f^{\prime}(w)\left\{w^{\prime 2}+\left(1+\mathcal{C} w^{\prime}\right)^{2}\right\}+1-\rho^{\prime}\left(\int_{-\pi}^{\pi} w \mathcal{C} w^{\prime} d t\right)\left(w^{\prime 2}+\left(1+\mathcal{C} w^{\prime}\right)^{2}-1\right) \equiv c .
\end{aligned}
$$

The proof is the same as that for (19), the only new ingredient being the following lemma.

Lemma 3. For all $w \in C_{2 \pi}^{\infty} \cap H$ and $W^{*}$ defined by (14),

$$
I^{\prime}(w) \phi=2 \int_{-\pi}^{\pi} w^{\prime} \phi^{\prime} d t=2 \int_{-\pi}^{\pi}\left\{w^{\prime \prime}+w^{\prime \prime} \mathcal{C} w^{\prime}-\mathcal{C}\left(w^{\prime} w^{\prime \prime}\right)\right\} \Im\left(\frac{\phi+i \mathcal{C} \phi}{W^{*}}\right) d t
$$

for all functions $\phi \in C_{2 \pi}^{\infty} \cap H$ with $(\phi+i \mathcal{C} \phi) / W^{*} \in C^{\infty}(\mathbb{R}, \mathbb{C})$.

Proof. We have

$$
\mathcal{C}\left(w^{\prime} w^{\prime \prime}-\mathcal{C} w^{\prime} \mathcal{C} w^{\prime \prime}\right)=w^{\prime} \mathcal{C} w^{\prime \prime}+w^{\prime \prime} \mathcal{C} w^{\prime}
$$

and

$$
\Re\left(\frac{\phi+i \mathcal{C} \phi}{W^{*}}\right)=-\mathcal{C} \Im\left(\frac{\phi+i \mathcal{C} \phi}{W^{*}}\right),
$$

since $(\phi+i \mathcal{C} \phi) / W^{*}$ has a holomorphic extension to the unit disc in the complex plane which is zero at 0 . Now

$$
I(w)=\int_{-\pi}^{\pi} \frac{1}{2}\left(\left|W^{*}\right|^{2}-1\right) d t
$$


and so

$$
\begin{aligned}
I^{\prime}(w) \phi & =\int_{-\pi}^{\pi} \Re\left(\overline{W^{*}}\left(\phi^{\prime}+i \mathcal{C} \phi^{\prime}\right)\right) d t \\
& =-\int_{-\pi}^{\pi} \Re\left(\overline{W^{* \prime}}(\phi+i \mathcal{C} \phi)\right) d t=-\int_{-\pi}^{\pi} \Re\left(W^{*} \overline{W^{*}} \frac{\phi+i \mathcal{C} \phi}{W^{*}}\right) d t \\
& =-\int_{-\pi}^{\pi} \Re\left(W^{*} \overline{W^{*}}\right) \Re\left(\frac{\phi+i \mathcal{C} \phi}{W^{*}}\right)-\Im\left(W^{*} \overline{W^{*}}\right) \Im\left(\frac{\phi+i \mathcal{C} \phi}{W^{*}}\right) d t \\
& =\int_{-\pi}^{\pi} \Re\left(W^{*} \overline{W^{*}}\right) \mathcal{C}\left(\Im\left(\frac{\phi+i \mathcal{C} \phi}{W^{*}}\right)\right)+\Im\left(W^{*} \overline{W^{*}}\right) \Im\left(\frac{\phi+i \mathcal{C} \phi}{W^{*}}\right) d t \\
& =\int_{-\pi}^{\pi}\left(-\mathcal{C}\left(\Re\left(W^{*} \overline{W^{*}}\right)\right)+\Im\left(W^{*} \overline{W^{*}}\right)\right) \Im\left(\frac{\phi+i \mathcal{C} \phi}{W^{*}}\right) d t \\
& =-\int_{-\pi}^{\pi}\left(\mathcal{C}\left(w^{\prime} w^{\prime \prime}+\left(1+\mathcal{C} w^{\prime}\right) \mathcal{C} w^{\prime \prime}\right)+w^{\prime} \mathcal{C} w^{\prime \prime}-w^{\prime \prime}\left(1+\mathcal{C} w^{\prime}\right)\right) \Im\left(\frac{\phi+i \mathcal{C} \phi}{W^{*}}\right) d t \\
& =\int_{-\pi}^{\pi}\left(2 w^{\prime \prime}-\mathcal{C}\left(w^{\prime} w^{\prime \prime}+\mathcal{C} w^{\prime} \mathcal{C} w^{\prime \prime}\right)-w^{\prime} \mathcal{C} w^{\prime \prime}+w^{\prime \prime} \mathcal{C} w^{\prime}\right) \Im\left(\frac{\phi+i \mathcal{C} \phi}{W^{*}}\right) d t \\
& =2 \int_{-\pi}^{\pi}\left\{w^{\prime \prime}+w^{\prime \prime} \mathcal{C} w^{\prime}-\mathcal{C}\left(w^{\prime} w^{\prime \prime}\right)\right\} \Im\left(\frac{\phi+i \mathcal{C} \phi}{W^{*}}\right) d t .
\end{aligned}
$$

This completes the proof.

\section{$5 \quad$ A priori estimates}

The mountain-pass lemma will be applied to $\mathcal{J}_{\epsilon}$. To understand the convergence of the critical points as $\epsilon \rightarrow 0$, we need a priori estimates that are independent of $\epsilon$. First we recall a useful lemma from [22].

Lemma 4. If $w \in W_{2 \pi}^{1,2}$ and if $h \in C^{\infty}(\mathbb{R})$ is convex on the range of $w$, then

$$
h^{\prime}(w(t)) \mathcal{C} w^{\prime}(t)-\mathcal{C}\left(h^{\prime}(w) w^{\prime}\right)(t) \geq 0
$$

almost everywhere and therefore

$$
\int_{-\pi}^{\pi} h^{\prime}(w(t)) \mathcal{C} w^{\prime}(t) d t \geq 0
$$

Proof.

$$
\begin{aligned}
h^{\prime}(w(t)) \mathcal{C} w^{\prime}(t) & -\mathcal{C}\left(h^{\prime}(w) w^{\prime}\right)(t) \\
& =\frac{1}{2 \pi} \int_{-\pi}^{\pi} \frac{\left(h^{\prime}(w(t))-h^{\prime}(w(s))\right) w^{\prime}(s)}{\tan ((t-s) / 2)} d s \\
& =\frac{1}{2 \pi} \int_{-\pi}^{\pi} \frac{(d / d s)\left(h(w(t))+h^{\prime}(w(t))(w(s)-w(t))-h(w(s))\right)}{\tan ((t-s) / 2)} d s \\
& =-\frac{1}{4 \pi} \int_{-\pi}^{\pi} \frac{h(w(t))+h^{\prime}(w(t))(w(s)-w(t))-h(w(s))}{\sin ^{2}((t-s) / 2)} d s \geq 0
\end{aligned}
$$

almost everywhere. (The integrand is negative since $h$ is convex.) 
Here is our first result giving an a priori estimate:

Proposition 5. If $w \in C_{2 \pi}^{\infty}$ satisfies $\int_{-\pi}^{\pi} w \mathcal{C} w^{\prime} d t<\pi R^{2}$ and (21) with $\epsilon>0$, then

$$
0 \leq \int_{-\pi}^{\pi} f^{\prime}(w) \mathcal{C} w^{\prime} d t \leq \int_{-\pi}^{\pi}-f^{\prime}(w) d t
$$

and

$$
\left\|w^{\prime}\right\|_{L_{2 \pi}^{2}}^{2} \leq \frac{\sup \left\{f^{\prime \prime}\right\} \int_{-\pi}^{\pi} w \mathcal{C} w^{\prime} d t}{2 \inf \left\{-f^{\prime}\right\}+2 \rho^{\prime}\left(\int_{-\pi}^{\pi} w \mathcal{C} w^{\prime} d t\right)}
$$

provided that $\inf \left\{-f^{\prime}\right\}>0$, sup $f^{\prime \prime}<\infty$ and $\rho^{\prime} \geq 0$ (this also holds for $\epsilon=0$ and $w \in H$ ).

Proof. Since $\epsilon \geq 0, \rho^{\prime} \geq 0$ and $\int_{-\pi}^{\pi} w^{\prime} \mathcal{C} w^{\prime \prime} d t \geq 0$, we deduce from (22)

$$
\int_{-\pi}^{\pi}-f^{\prime}(w)\left\{\left(\mathcal{C} w^{\prime}\right)^{2}+w^{\prime 2}\right\} d t+2 \rho^{\prime}\left(\int_{-\pi}^{\pi} w \mathcal{C} w^{\prime} d t\right)\left(\int_{-\pi}^{\pi} w^{\prime 2} d t\right) \leq \int_{-\pi}^{\pi} f^{\prime}(w) \mathcal{C} w^{\prime} d t .
$$

It follows from this and Hölder's inequality that

$$
\frac{\left(\int_{-\pi}^{\pi} f^{\prime}(w) \mathcal{C} w^{\prime} d t\right)^{2}}{\int_{-\pi}^{\pi}-f^{\prime}(w) d t} \leq \int_{-\pi}^{\pi}-f^{\prime}(w)\left(\mathcal{C} w^{\prime}\right)^{2} d t \leq \int_{-\pi}^{\pi} f^{\prime}(w) \mathcal{C} w^{\prime} d t
$$

and the first assertion follows. That

$$
\left\|w^{\prime}\right\|_{L_{2 \pi}^{2}}^{2} \leq \frac{\int_{-\pi}^{\pi} f^{\prime}(w) \mathcal{C} w^{\prime} d t}{2 \inf \left\{-f^{\prime}\right\}+2 \rho^{\prime}\left(\int_{-\pi}^{\pi} w \mathcal{C} w^{\prime} d t\right)}
$$

follows immediately from (23). We then apply Lemma 4 with $h^{\prime}(s)=s \sup f^{\prime \prime}-f^{\prime}(s)$.

Let $\widehat{H}$ denote the completion of $H$ with respect to the metric defined by the inner product

$$
\langle u, v\rangle_{\frac{1}{2}}=\sum_{n \geq 1} n u_{n} v_{n}
$$

in which $u_{k}, v_{k}$ denote the Fourier coefficients of $u, v \in H$ with respect to the orthonormal basis $\left\{\pi^{-\frac{1}{2}} \cos n t: n \in \mathbb{N}\right\}$. Thus $\widehat{H}$ is a subspace of the fractional Sobolev space defined in (3). Clearly $\widehat{H}$ is a Hilbert subspace of even functions with zero mean in $L_{2 \pi}^{2}$ and

$$
\langle u, v\rangle_{\frac{1}{2}}=\int_{-\pi}^{\pi} u \mathcal{C} v^{\prime} d t=\int_{-\pi}^{\pi} v \mathcal{C} u^{\prime} d t, \quad u, v \in H
$$

Now for $\epsilon>0$ define a new inner product on $H$ by

$$
\langle u, v\rangle_{\epsilon}=2 \int_{-\pi}^{\pi} \epsilon u^{\prime} v^{\prime}+u \mathcal{C} v^{\prime} d t
$$

Let $w \in C_{2 \pi}^{\infty}$ be a solution of $(21)$ (with $\int_{-\pi}^{\pi} w \mathcal{C} w^{\prime} d t<\pi R^{2}$ ). It follows that there exists a linear operator $L_{\epsilon}: H \rightarrow H$ such that

$$
\mathcal{J}_{\epsilon}^{\prime \prime}(w)(u, v)=\left\langle L_{\epsilon} u, v\right\rangle_{\epsilon}, \quad u, v \in H
$$


Assume that $\mu$ is a non-positive eigenvalue of $L_{\epsilon}$ :

$$
L_{\epsilon} u=\mu u, \quad u \in H \backslash\{0\}, \quad \mu \leq 0 .
$$

Hence

$$
\mathcal{J}_{\epsilon}^{\prime \prime}(w)(u, v)=\mu\langle u, v\rangle_{\epsilon}, \quad v \in H .
$$

Proposition 6. If $\inf \left\{-f^{\prime}(w(t))\right\}>0, \rho^{\prime} \geq 0$ and $\rho^{\prime \prime} \geq 0$, then

$$
2\left(\inf \left\{-f^{\prime}(w(t))\right\}-\mu\right) \int_{-\pi}^{\pi} u \mathcal{C} u^{\prime} d t \leq \sup \left\{\left|f^{\prime \prime}(w(t))\right|\right\}\left(\sqrt{2 \pi}+2\left\|w^{\prime}\right\|_{L_{2 \pi}^{2}}\right)\left\|u^{2}\right\|_{L_{2 \pi}^{2}} .
$$

Remark.The right-hand side can be further estimated thanks to Proposition 5. If $\inf \left\{-f^{\prime}\right\}>0, \rho^{\prime} \geq 0, \rho^{\prime \prime} \geq 0, R<\infty, \sup f^{\prime \prime}<\infty$ and $u$ is normalized by $\int_{-\pi}^{\pi} u \mathcal{C} u^{\prime} d t=1$, Proposition 6 then gives an upper bound on $|\mu|$ and a positive lower bound on $\left\|u^{2}\right\|_{L_{2 \pi}^{2}}$ that are independent of $\epsilon$.

Proof. Since $\mu \leq 0$ this follows from (12) because

$$
\begin{aligned}
2 \mu \int_{-\pi}^{\pi} u \mathcal{C} u^{\prime} d t & \geq 2 \mu \int_{-\pi}^{\pi} \epsilon u^{\prime 2}+u \mathcal{C} u^{\prime} d t=\mu\langle u, u\rangle_{\epsilon}=\mathcal{J}_{\epsilon}^{\prime \prime}(w)(u, u) \\
& =2 \epsilon \int_{-\pi}^{\pi}\left|u^{\prime}\right|^{2} d t-\int_{-\pi}^{\pi} u\left\{f^{\prime \prime}(w)\left(1+\mathcal{C} w^{\prime}\right) u+f^{\prime}(w) \mathcal{C} u^{\prime}+\mathcal{C}\left(f^{\prime}(w) u\right)^{\prime}\right\} d t \\
& +2 \rho^{\prime}\left(\int_{-\pi}^{\pi} w \mathcal{C} w^{\prime} d t\right) \int_{-\pi}^{\pi} u \mathcal{C} u^{\prime} d t+4 \rho^{\prime \prime}\left(\int_{-\pi}^{\pi} w \mathcal{C} w^{\prime} d t\right)\left(\int_{-\pi}^{\pi} u \mathcal{C} w^{\prime} d t\right)^{2} \\
& \geq-\int_{-\pi}^{\pi} u\left\{f^{\prime \prime}(w)\left(1+\mathcal{C} w^{\prime}\right) u+f^{\prime}(w) \mathcal{C} u^{\prime}+\mathcal{C}\left(f^{\prime}(w) u\right)^{\prime}\right\} d t \\
& =\int_{-\pi}^{\pi} 2\left\{-f^{\prime}(w)\right\}\left\{u \mathcal{C} u^{\prime}-\mathcal{C}\left(u u^{\prime}\right)\right\} d t-\int_{-\pi}^{\pi}\left\{f^{\prime \prime}(w)\left(1+\mathcal{C} w^{\prime}\right)+\mathcal{C}\left(f^{\prime}(w)\right)^{\prime}\right\} u^{2} \\
& \geq \int_{-\pi}^{\pi} 2\left\{-f^{\prime}(w)\right\}\left\{u \mathcal{C} u^{\prime}-\mathcal{C}\left(u u^{\prime}\right)\right\} d t \\
& -\sup \left\{\left|f^{\prime \prime}(w(t))\right|\right\}\left(\sqrt{2 \pi}+2\left\|w^{\prime}\right\|_{L_{2 \pi}^{2}}\right)\left\|u^{2}\right\|_{L_{2 \pi}^{2}} d t
\end{aligned}
$$

since $u \mathcal{C} u^{\prime}-\mathcal{C}\left(u u^{\prime}\right) \geq 0$ almost everywhere, by Lemma 4 .

\section{Application of the Mountain-Pass Lemma}

In this section we prove the existence of a saddle point of $\mathcal{J}$ restricted to $H$ if $\mathcal{J}$ has the right mountain-pass structure. For a correct choice of the parameters, the critical point then gives a smooth solution of (7) (see next section). It is convenient to distinguish the linear and nonlinear parts of $f$. To do so we write

$$
f(x):=\lambda g(x)-x, \quad x \in \mathbb{R},
$$

where $\lambda \in(0,1)$ is fixed, $g: \mathbb{R} \rightarrow \mathbb{R}$ is a smooth function with

$$
g(0)=0, \quad g^{\prime}(0)=0, \quad g^{\prime \prime}(0)=2,
$$




$$
\sup _{x \in \mathbb{R}} g^{\prime}(x) \leq 2 M \quad \text { and } \quad \sup _{x \in \mathbb{R}} g^{\prime \prime}(x)<\infty
$$

for some constant $M<1 /(2 \lambda)$. The function $f$ thus satisfies

$$
\inf \left\{-f^{\prime}\right\}>0 \text { and } \sup f^{\prime \prime}<\infty \text {. }
$$

Now let $0<R<\infty$ and let $\rho:\left[0, \pi R^{2}\right) \rightarrow \mathbb{R}$ be $C^{\infty}$ and convex, with $\rho(0)=0, \rho^{\prime}(0) \geq 0$ and

$$
\rho(s) \rightarrow+\infty \quad \text { as } \quad s \rightarrow \pi R^{2}
$$

Note that

$$
\mathcal{J}(w) \rightarrow \infty \text { as } \int_{-\pi}^{\pi} w \mathcal{C} w^{\prime} d t \nearrow \pi R^{2}
$$

since

$$
\begin{aligned}
\mathcal{J}(w) & =\int_{-\pi}^{\pi}\left\{(w-\lambda g(w)) \mathcal{C} w^{\prime}-\lambda g(w)\right\} d t+\rho\left(\int_{-\pi}^{\pi} w \mathcal{C} w^{\prime} d t\right) \\
& \geq-\lambda \int_{-\pi}^{\pi} g(w) d t+\rho\left(\int_{-\pi}^{\pi} w \mathcal{C} w^{\prime} d t\right) \\
& \geq-\lambda\left(\sup g^{\prime \prime} / 2\right)\|w\|_{L_{2 \pi}^{2}}^{2}+\rho\left(\int_{-\pi}^{\pi} w \mathcal{C} w^{\prime} d t\right) \\
& \geq-\lambda\left(\sup g^{\prime \prime} / 2\right) \int_{-\pi}^{\pi} w \mathcal{C} w^{\prime} d t+\rho\left(\int_{-\pi}^{\pi} w \mathcal{C} w^{\prime} d t\right) \\
& \geq-\lambda\left(\sup g^{\prime \prime} / 2\right) \pi R^{2}+\rho\left(\int_{-\pi}^{\pi} w \mathcal{C} w^{\prime} d t\right)
\end{aligned}
$$

where the first inequality follows from Lemma 4 applied to $h^{\prime}(w)=-f(w)=w-\lambda g(w)$. The functional $\mathcal{J}$ is of class $C^{2}$ on $H$, but it is not of class $C^{2}$ on $\widehat{H}$ (see $(24)$ for the definition of $\widehat{H}$ ). However, for all $w \in H$, the second derivative $\mathcal{J}^{\prime \prime}(w): H \times H \rightarrow \mathbb{R}$ has a continuous extension $\widehat{\mathcal{J}}^{\prime \prime}(w): \widehat{H} \times \widehat{H} \rightarrow \mathbb{R}$. Indeed, for all $w \in H,(12)$ implies that

$$
\begin{aligned}
\left|\mathcal{J}^{\prime \prime}(w)(\phi, \phi)\right|= & \mid \int_{-\pi}^{\pi} 2\left(1-\lambda g^{\prime}(w)\right) \phi \mathcal{C} \phi^{\prime}-\lambda g^{\prime \prime}(w)\left(1+\mathcal{C} w^{\prime}\right) \phi^{2} d t \\
& +2 \rho^{\prime}\left(\int_{-\pi}^{\pi} w \mathcal{C} w^{\prime} d t\right) \int_{-\pi}^{\pi} \phi \mathcal{C} \phi^{\prime} d t+4 \rho^{\prime \prime}\left(\int_{-\pi}^{\pi} w \mathcal{C} w^{\prime} d t\right)\left(\int_{-\pi}^{\pi} \phi \mathcal{C} w^{\prime} d t\right)^{2} \mid \\
= & \mid 2 \int_{-\pi}^{\pi}\left(1-\lambda g^{\prime}(w)\right)\left\{\phi \mathcal{C} \phi^{\prime}-\mathcal{C}\left(\phi \phi^{\prime}\right)\right\} d t \\
& +\int_{-\pi}^{\pi}\left\{\mathcal{C}\left(1-\lambda g^{\prime}(w)\right)^{\prime}-\lambda g^{\prime \prime}(w)\left(1+\mathcal{C} w^{\prime}\right)\right\} \phi^{2} d t \\
& +2 \rho^{\prime}\left(\int_{-\pi}^{\pi} w \mathcal{C} w^{\prime} d t\right) \int_{-\pi}^{\pi} \phi \mathcal{C} \phi^{\prime} d t+4 \rho^{\prime \prime}\left(\int_{-\pi}^{\pi} w \mathcal{C} w^{\prime} d t\right)\left(\int_{-\pi}^{\pi} \phi \mathcal{C} w^{\prime} d t\right)^{2} \mid \\
\leq & K\|\phi\|_{\frac{1}{2}}^{2}
\end{aligned}
$$

for some constant $K>0$ and all $\phi \in H$, because $\phi \mathcal{C} \phi^{\prime}-\mathcal{C}\left(\phi \phi^{\prime}\right) \geq 0$ almost everywhere by Lemma 4. Applying the Cauchy-Schwartz inequality to the positive symmetric bilinear form $\left(\phi_{1}, \phi_{2}\right) \rightarrow \mathcal{J}^{\prime \prime}(w)\left(\phi_{1}, \phi_{2}\right)+K\left\langle\phi_{1}, \phi_{2}\right\rangle_{\frac{1}{2}}$, we get

$$
\left|\mathcal{J}^{\prime \prime}(w)\left(\phi_{1}, \phi_{2}\right)\right| \leq \mathrm{Const}\left\|\phi_{1}\right\|_{\frac{1}{2}}\left\|\phi_{2}\right\|_{\frac{1}{2}}
$$


for all $\phi_{1}, \phi_{2} \in H$. Therefore $\mathcal{J}^{\prime \prime}(w)\left(\phi_{1}, \phi_{2}\right)$ and $\mathcal{J}^{\prime \prime}(w)(\phi, \phi)$ can be extended by continuity to all $\phi_{1}, \phi_{2}, \phi \in \widehat{H}$.

Theorem 7. Suppose that there exists $u_{*} \in H$ such that

$$
\int_{-\pi}^{\pi} u_{*} \mathcal{C} u_{*}^{\prime} d t<\pi R^{2} \text { and } \mathcal{J}\left(u_{*}\right)<0
$$

Then there exists a solution $w \in H$ of (18) such that

$$
\int_{-\pi}^{\pi} w \mathcal{C} w^{\prime} d t<\pi R^{2}, \quad 0 \leq \mathcal{J}(w) \leq \max _{0 \leq s \leq 1} \mathcal{J}\left(s u_{*}\right)
$$

and $\widehat{\mathcal{J}}^{\prime \prime}(w)$ is not positive definite:

$$
\widehat{\mathcal{J}}^{\prime \prime}(w)(\widehat{u}, \widehat{u}) \leq 0 \text { for some } \widehat{u} \in \widehat{H} \backslash\{0\}
$$

Since

$$
\widehat{\mathcal{J}}^{\prime \prime}(0)(u, u)=2\|u\|_{\frac{1}{2}}^{2}-2 \lambda \int_{-\pi}^{\pi} u^{2} d t>0
$$

for all $u \in \widehat{H} \backslash\{0\}$, it follows that $w \neq 0$.

Proof. The rest of this section is devoted to the proof. Recall that $H$ is compactly embedded in $L^{\infty}(\mathbb{R})$ and that, for $w(t)=\frac{1}{\sqrt{\pi}} \sum_{n \geq 1} w_{n} \cos n t \in H$,

$$
\int_{-\pi}^{\pi} w \mathcal{C} w^{\prime} d t=\sum_{n \geq 1} n w_{n}^{2} \geq\|w\|_{L_{2 \pi}^{2}}^{2}
$$

Since $\lambda \in(0,1)$, for $w \in H$ with $\|w\|_{H}$ sufficiently small,

$$
\mathcal{J}(w) \geq \int_{-\pi}^{\pi} w \mathcal{C} w^{\prime}-\lambda w^{2} d t-\lambda \int_{-\pi}^{\pi}\left\{g(w)-(1 / 2) g^{\prime \prime}(0) w^{2}+g(w) \mathcal{C} w^{\prime}\right\} d t \geq-K\|w\|_{H}^{3}
$$

for some $K>0$. Therefore, for any $\epsilon>0$ there exist $a_{\epsilon}, b_{\epsilon}>0$ such that

$$
\|w\|_{H}=a_{\epsilon} \text { implies that } \mathcal{J}_{\epsilon}(w) \geq b_{\epsilon} .
$$

Now we check that $\mathcal{J}_{\epsilon}$ satisfies the Palais-Smale condition. Suppose that $\left\{w_{k}\right\} \subset H$ is such that $\int_{-\pi}^{\pi} w_{k} \mathcal{C} w_{k}^{\prime} d t<\pi R^{2}, \sup _{k}\left|\mathcal{J}_{\epsilon}\left(w_{k}\right)\right|<\infty$ and $\mathcal{J}_{\epsilon}^{\prime}\left(w_{k}\right) \rightarrow 0$ in $H^{*}$ as $k \rightarrow \infty$. From

$$
\begin{aligned}
\mathcal{J}_{\epsilon}\left(w_{k}\right) & \geq \int_{-\pi}^{\pi} \epsilon\left|w_{k}^{\prime}\right|^{2}-\lambda g\left(w_{k}\right) d t+\rho\left(\int_{-\pi}^{\pi} w_{k} \mathcal{C} w_{k}^{\prime} d t\right) \\
& \geq \epsilon\left\|w_{k}\right\|_{H}^{2}-\lambda\left(\sup g^{\prime \prime} / 2\right)\left\|w_{k}\right\|_{L_{2 \pi}^{2}}^{2} \\
& \geq \epsilon\left\|w_{k}\right\|_{H}^{2}-\lambda\left(\sup g^{\prime \prime} / 2\right) \int_{-\pi}^{\pi} w_{k} \mathcal{C} w_{k}^{\prime} d t \\
& \geq \epsilon\left\|w_{k}\right\|_{H}^{2}-\lambda\left(\sup g^{\prime \prime} / 2\right) \pi R^{2}
\end{aligned}
$$


it follows that $\left\{w_{k}\right\}$ is bounded in $H$. Extracting a subsequence we may assume that $w_{k} \rightarrow w$ in $H$ as $k \rightarrow \infty$, with $\int_{-\pi}^{\pi} w \mathcal{C} w^{\prime} d t<\pi R^{2}$. Now $\mathcal{J}_{\epsilon}^{\prime}\left(w_{k}\right) \rightarrow 0$ in $H^{*}$ as $k \rightarrow \infty$, where

$$
\begin{aligned}
\mathcal{J}_{\epsilon}^{\prime}(w) v=\int_{-\pi}^{\pi}\left(2 \epsilon w^{\prime} v^{\prime}-\lambda g^{\prime}(w) v+(w-\lambda g(w)) \mathcal{C} v^{\prime}\right. & \left.+\left(1-\lambda g^{\prime}(w)\right) v \mathcal{C} w^{\prime}\right) d t \\
& +2 \rho^{\prime}\left(\int_{-\pi}^{\pi} w \mathcal{C} w^{\prime} d t\right) \int_{-\pi}^{\pi} v \mathcal{C} w^{\prime} d t
\end{aligned}
$$

Hence

$$
\begin{aligned}
\left(\mathcal{J}_{\epsilon}^{\prime}\left(w_{k}\right)\right. & \left.-\mathcal{J}_{\epsilon}^{\prime}\left(w_{l}\right)\right)\left(w_{k}-w_{l}\right)=\int_{-\pi}^{\pi}\left\{2 \epsilon\left(w_{k}^{\prime}-w_{l}^{\prime}\right)^{2}-\lambda\left(g^{\prime}\left(w_{k}\right)-g^{\prime}\left(w_{l}\right)\right)\left(w_{k}-w_{l}\right)\right. \\
& +\left(w_{k}-w_{l}-\lambda\left(g^{\prime}\left(w_{k}\right)-g^{\prime}\left(w_{l}\right)\right) \mathcal{C}\left(w_{k}^{\prime}-w_{l}^{\prime}\right)\right. \\
& \left.+\left(w_{k}-w_{l}\right) \mathcal{C}\left(w_{k}^{\prime}-w_{l}^{\prime}\right)-\lambda\left(g^{\prime}\left(w_{k}\right) \mathcal{C} w_{k}^{\prime}-g^{\prime}\left(w_{l}\right) \mathcal{C} w_{l}^{\prime}\right)\left(w_{k}-w_{l}\right)\right\} d t \\
& +2 \int_{-\pi}^{\pi}\left\{\rho^{\prime}\left(\int_{-\pi}^{\pi} w_{k} \mathcal{C} w_{k}^{\prime} d t\right) \mathcal{C} w_{k}^{\prime}-\rho^{\prime}\left(\int_{-\pi}^{\pi} w_{l} \mathcal{C} w_{l}^{\prime} d t\right) \mathcal{C} w_{l}^{\prime}\right\}\left(w_{k}-w_{l}\right) d t \rightarrow 0
\end{aligned}
$$

as $k, l \rightarrow \infty$. Since $w_{k} \rightarrow w$ uniformly and $w_{k}^{\prime} \rightarrow w^{\prime}$ in $L_{2 \pi}^{2}$ it is immediate that

$$
\int_{-\pi}^{\pi} 2 \epsilon\left(w_{k}^{\prime}-w_{l}^{\prime}\right)^{2} d t \rightarrow 0,
$$

as $k, l \rightarrow \infty$. Hence $\left\{w_{k}\right\}$ is convergent to $w$ in $H$. This shows that $\mathcal{J}_{\epsilon}$ satisfies the Palais-Smale condition.

Since the functional $\mathcal{J}_{\epsilon}$ on $H$ has mountain-pass geometry at $0 \in H$ and satisfies the Palais-Smale condition, there exists a critical point $w_{\epsilon}$ of $\mathcal{J}_{\epsilon}$ on $H$ with the property that

$$
\mathcal{J}_{\epsilon}\left(w_{\epsilon}\right)=\inf _{\gamma \in \Gamma} \sup _{u \in \gamma} \mathcal{J}_{\epsilon}(u) \leq \max _{0 \leq s \leq 1} \mathcal{J}_{\epsilon}\left(s u_{*}\right),
$$

where $\Gamma$ denotes the set of continuous paths in $H$ joining 0 and $u_{*}$ [2].

The next step is to let $\epsilon \rightarrow 0$ and for this we need an a priori bound. Since $w_{\epsilon}$ is a critical point of $\mathcal{J}_{\epsilon}$, Proposition 5 gives

$$
\left\|w_{\epsilon}^{\prime}\right\|_{L_{2 \pi}^{2}}^{2} \leq \frac{\sup f^{\prime \prime}}{2 \inf \left\{-f^{\prime}\right\}} \pi R^{2}
$$

and therefore $w_{\epsilon}$ is bounded in $H$ uniformly in $\epsilon$. By taking a sequence of $\epsilon \rightarrow 0$, we may suppose that $w_{\epsilon} \rightarrow w$ as $\epsilon \rightarrow 0$. Since $\mathcal{J}_{\epsilon}^{\prime}\left(w_{\epsilon}\right) v=0$, taking the limit as $\epsilon \rightarrow 0$ in (28) yields that for all $v \in H$,

$$
\begin{aligned}
& 0=\mathcal{J}^{\prime}(w) v \\
= & \int_{-\pi}^{\pi}\left(-\lambda g^{\prime}(w) v+\left(1-\lambda g^{\prime}(w)\right) v \mathcal{C} w^{\prime}+(w-\lambda g(w)) \mathcal{C} v^{\prime}\right) d t+2 \rho^{\prime}\left(\int_{-\pi}^{\pi} w \mathcal{C} w^{\prime} d t\right) \int_{-\pi}^{\pi} v \mathcal{C} w^{\prime} d t
\end{aligned}
$$

and hence that $w \in H$ satisfies (18). Moreover

$$
0 \leq \mathcal{J}(w) \leq \max _{0 \leq s \leq 1} \mathcal{J}\left(s u_{*}\right) .
$$


Recall space $\widehat{H}$ in (24), the inner product on $H$ for $\epsilon>0$ in (25), and the linear operator $L_{\epsilon}: H \rightarrow H$ such that

$$
\mathcal{J}_{\epsilon}^{\prime \prime}\left(w_{\epsilon}\right)(u, v)=\left\langle L_{\epsilon} u, v\right\rangle_{\epsilon}, \quad u, v \in H
$$

Since $w_{\epsilon}$ is a mountain-pass critical point of $\mathcal{J}_{\epsilon}$, the bilinear form $\mathcal{J}_{\epsilon}^{\prime \prime}\left(w_{\epsilon}\right)(v, v)$ is nonpositive for $v$ in a non-trivial subspace of $H$ (see [17], [16, Ch. 6]). Therefore the selfadjoint operator $L_{\epsilon}$ has a non-positive eigenvalue $\mu_{\epsilon}: L_{\epsilon} u_{\epsilon}=\mu_{\epsilon} u_{\epsilon}$ for some $u_{\epsilon} \in H \backslash\{0\}$. Hence, by Proposition 6,

$$
2\left(\inf \left\{-f^{\prime}\right\}-\mu_{\epsilon}\right) \int_{-\pi}^{\pi} u_{\epsilon} \mathcal{C} u_{\epsilon}^{\prime} d t \leq \text { const }\left\|u_{\epsilon}^{2}\right\|_{L_{2 \pi}^{2}},
$$

where the constant is independent of $\epsilon$. Now normalize $u_{\epsilon}$ such that $\left\|u_{\epsilon}\right\|_{\frac{1}{2}}=1$. Then, since $\widehat{H}$ is continuously embedded in $L^{4}(-\pi, \pi)$, there is a constant $K_{1}$ such that $\left\|u_{\epsilon}^{2}\right\|_{L_{2 \pi}^{2}} \leq$ $K_{1}$. Since $\mu_{\epsilon} \leq 0$ it follows that

$$
-\mu_{\epsilon} \leq \text { const and } 1=\left\|u_{\epsilon}\right\|_{\frac{1}{2}}^{2} \leq \text { const }\left\|u_{\epsilon}^{2}\right\|_{L_{2 \pi}^{2}} .
$$

Suppose that $u_{\epsilon} \rightarrow \widehat{u}$ in $\widehat{H}$ and $\mu_{\epsilon} \rightarrow \hat{\mu} \in(-\infty, 0]$ as $\epsilon \rightarrow 0$. Since $u_{\epsilon}^{2}$ converges strongly in $L_{2 \pi}^{2}$ to $\widehat{u}^{2}$, it follows that $\widehat{u} \neq 0$.

Since, by assumption, $w_{\epsilon} \rightarrow w$ in $H$, it follows from (26) that for all smooth $v \in H$

$$
\begin{aligned}
2 \hat{\mu} \int_{-\pi}^{\pi} \widehat{u} \mathcal{C} v^{\prime} d t & =\int_{-\pi}^{\pi}\left(1-\lambda g^{\prime}(w)\right) \widehat{u} \mathcal{C} v^{\prime}+\widehat{u} \mathcal{C}\left\{\left(1-\lambda g^{\prime}(w)\right) v\right\}^{\prime}-\lambda\left(1+\mathcal{C} w^{\prime}\right) g^{\prime \prime}(w) \widehat{u} v d t \\
& +2 \rho^{\prime}\left(\int_{-\pi}^{\pi} w \mathcal{C} w^{\prime} d t\right) \int_{-\pi}^{\pi} \widehat{u} \mathcal{C} v^{\prime} d t+4 \rho^{\prime \prime}\left(\int_{-\pi}^{\pi} w \mathcal{C} w^{\prime} d t\right) \int_{-\pi}^{\pi} \widehat{u} \mathcal{C} w^{\prime} d t \int_{-\pi}^{\pi} \widehat{v} \mathcal{C} w^{\prime} d t \\
& =\widehat{\mathcal{J}}^{\prime \prime}(w)(\widehat{u}, v) .
\end{aligned}
$$

This equality can be extended by continuity to all $v \in \widehat{H}$ and, setting $v=\widehat{u}$,

$$
\widehat{\mathcal{J}}^{\prime \prime}(w)(\widehat{u}, \widehat{u})=2 \hat{\mu}\|\widehat{u}\|_{\frac{1}{2}}^{2} \leq 0 .
$$

This completes the proof of Theorem 7

\section{Small water waves}

¿From here on we suppose, in addition, that

$$
g(x)=x^{2} \text { for all } x \leq m \quad \text { and } \quad g^{\prime \prime}(x) \leq 2 \text { for all } x \in \mathbb{R}
$$

where $0<m<M$, and

$$
\rho(s)=\frac{\lambda}{2 \pi} s^{2} \quad \text { for } 0 \leq s \leq \pi r^{2} \quad \text { and } \quad \rho^{\prime}(s) \geq \frac{\lambda}{\pi} s \quad \text { for } 0 \leq s<\pi R^{2},
$$

where $0<r<R<\infty$. Moreover we assume that $M<1 / 2$ (which implies $M<1 /(2 \lambda$ ) uniformly in $\lambda \in(0,1)$ ). We easily get the following particular case of Theorem 7 . 
Theorem 8. Suppose that there exists $u_{*} \in H$ such that

1. $\max u_{*} \leq m$,

2. $\int_{-\pi}^{\pi} u_{*} \mathcal{C} u_{*}^{\prime} d t \leq \pi r^{2}$,

3. $\mathcal{J}\left(u_{*}\right)=\int_{-\pi}^{\pi}\left\{u_{*} \mathcal{C} u_{*}^{\prime}-\lambda u_{*}^{2}\left(1+\mathcal{C} u_{*}^{\prime}\right)\right\} d t+\lambda(2 \pi)^{-1}\left(\int_{-\pi}^{\pi} u_{*} \mathcal{C} u_{*}^{\prime} d t\right)^{2}<0$.

Then there exists a solution $w \in H \backslash\{0\}$ of (18) which satisfies (27).

The existence of $u_{*}$ is an important issue, as well as whether the estimates (27) ensure that $\max w \leq m$ for right choices of $r$ and $R$, in which case $w \neq 0$ is smooth and gives rise to a Stokes wave. The fact that $w$ is smooth is proved in $[8,24]$ by a bootstrap on the equation

$$
\mathcal{C} w^{\prime}=-\frac{c-1-f^{\prime}(w)+f^{\prime}(w) \mathcal{C} w^{\prime}-\mathcal{C}\left(f^{\prime}(w) w^{\prime}\right)}{-2 f^{\prime}(w)+2 \rho^{\prime}\left(\int_{-\pi}^{\pi} w \mathcal{C} w^{\prime} d t\right)},
$$

thanks to the property $\inf \left\{-f^{\prime}(w)\right\}=\inf \left\{1-\lambda g^{\prime}(w)\right\}>0$ and to the fact that $f^{\prime}(w) \mathcal{C} w^{\prime}-$ $\mathcal{C}\left(f^{\prime}(w) w^{\prime}\right)=2 \lambda\left(w \mathcal{C} w^{\prime}-\mathcal{C}\left(w w^{\prime}\right)\right)$ is more regular than first expected.

For $u_{*}$, we choose $u_{*}(t)=a(\cos t+k \cos 2 t)$, where $a, k>0$. Then

$$
\left\|u_{*}\right\|_{L^{\infty}(\mathbb{R})}=a(1+k) \text { and } \int_{-\pi}^{\pi} u_{*} \mathcal{C} u_{*}^{\prime} d t=\pi a^{2}\left(1+2 k^{2}\right) .
$$

We first make rough estimates to give an idea of the method. We fix $m>0$, let $a(1+k) \leq$ $m$ and find

$$
\mathcal{J}\left(u_{*}\right)=\pi a^{2}\left\{1+2 k^{2}-\lambda\left(1+k^{2}+2 a k\right)\right\}+\lambda(2 \pi)^{-1}\left\{\pi a^{2}\left(1+2 k^{2}\right)\right\}^{2} .
$$

We choose $k$ of the same order as $a$ and $1-\lambda \in(0,1)$ of at most the same order as $a^{2}$. In order that $\mathcal{J}\left(u_{*}\right)<0$ for small $a$, we have therefore to check (keeping only the terms of order at most 2 in $a$ )

$$
1-\lambda+k^{2}-2 a k+(1 / 2) a^{2}<0,
$$

which holds if we choose $k=a$ and $\lambda \in\left[1-(1 / 4) a^{2}, 1\right)$.

Hence the three hypotheses of Theorem 8 are verified if $a$ is small enough, $\lambda \in[1-$ $\left.(1 / 4) a^{2}, 1\right)$ and $r>0$ is chosen such that

$$
\pi r^{2}=\int_{-\pi}^{\pi} u_{*} \mathcal{C} u_{*}^{\prime} d t=\pi a^{2}\left(1+2 a^{2}\right) .
$$

By Proposition 5 with $\epsilon=0$, we can bound $\int_{-\pi}^{\pi} w^{\prime 2} d t$ by a function of $R$, $\left(\inf \left\{-f^{\prime}\right\}\right)^{-1}<$ $(1-2 M)^{-1}<\infty$ and $\sup f^{\prime \prime}<2$. On the other hand, taking $R=2 r$, we also have

$$
\int_{-\pi}^{\pi} w \mathcal{C} w^{\prime} d t<4 \pi a^{2}\left(1+2 a^{2}\right) \rightarrow 0
$$

as $a \rightarrow 0$. Hence standard interpolation inequalities give that $\max |w|$ converges to 0 as $a \rightarrow 0$, which shows that $\max w \leq m$ for small enough $a$. Thus we have proved by an infinite-dimensional variational argument the existence of small periodic water waves. 
In what follows, we give better estimates on the region of existence of periodic water waves. We suppose again that $a(1+k) \leq m$. Then $g\left(u_{*}\right)=u_{*}^{2}$ and with $r=a \sqrt{1+2 k^{2}}$,

$$
\mathcal{J}\left(u_{*}\right)=\pi a^{2}\left\{1-\lambda+(2-\lambda) k^{2}-\frac{2 \lambda r k}{\sqrt{1+2 k^{2}}}+(\lambda / 2) r^{2}\left(1+2 k^{2}\right)\right\} .
$$

So $\mathcal{J}\left(u_{*}\right)<0$, and thus the three hypotheses of Theorem 8 are verified, if the two conditions below hold:

$$
\begin{gathered}
1-\lambda+(2-\lambda) k^{2}-\frac{2 \lambda r k}{\sqrt{1+2 k^{2}}}+(\lambda / 2) r^{2}\left(1+2 k^{2}\right)<0 \\
r \frac{1+k}{\sqrt{1+2 k^{2}}} \leq m .
\end{gathered}
$$

Theorem 8 provides us with a non-trivial solution $w$ of (18) and (27), which therefore satisfies, by Proposition 5,

$$
\left\|w^{\prime}\right\|_{L_{2 \pi}^{2}}^{2} \leq \frac{\lambda \pi R^{2}}{1-2 \lambda M+\lambda R^{2}} .
$$

But $[w]=0$. So, writing $w(t)=\sum_{n \geq 1} a_{n} \cos (n t)$, we have, for all $t$,

$$
\begin{aligned}
|w(t)| & \leq \sum_{n \geq 1}\left|a_{n}\right| \leq\left(\sum_{n \geq 1} \pi n^{2}\left|a_{n}\right|^{2}\right)^{1 / 2}\left(\pi^{-1} \sum_{n \geq 1} \frac{1}{n^{2}}\right)^{1 / 2} \\
& =\sqrt{\frac{\pi}{6}}\left\|w^{\prime}\right\|_{L_{2 \pi}^{2}} .
\end{aligned}
$$

Now, $w$ gives rise to a water wave if $w(t) \leq m$ for all $t$, so a sufficient condition is

$$
\frac{\pi^{2} \lambda R^{2}}{6\left(1-2 \lambda M+\lambda R^{2}\right)} \leq m^{2} .
$$

Since we can take $M$ arbitrarily close to $m$, and $R$ arbitrarily close to $r$, it is sufficient to check the condition

$$
\frac{\pi^{2} \lambda r^{2}}{6\left(1-2 \lambda m+\lambda r^{2}\right)}<m^{2}
$$

which can be rewritten

$$
r^{2}<\frac{(1-2 \lambda m) m^{2}}{\lambda\left(\frac{\pi^{2}}{6}-m^{2}\right)}
$$

if $m^{2}<\pi^{2} / 6$. We recall here the two other conditions (29) and (30). Take $m=0.345$, $r=0.158, k=0.142, \lambda=0.99:$ the three conditions above are fulfilled, and we get a solution of (7) in which $w$ and $\lambda$ are replaced by $w_{*}=w-\frac{c}{2 \lambda}$ and

$$
\lambda_{*}=\frac{0.99}{1+\rho^{\prime}\left(\int_{-\pi}^{\pi} w \mathcal{C} w^{\prime} d t\right)-c} \leq 0.99 .
$$




\section{References}

[1] H. Amann and E. Zehnder, Nontrivial solutions for a class of nonresonance problems and applications to nonlinear differential equations, Ann. Scuola Norm. Sup. Pisa Cl. Sci. IV 7(4) (1980), 539-603.

[2] A. Ambrosetti and P. H. Rabinowitz, Dual variational methods in critical point theory and applications, J. Funct. Anal. 14 (1973), 349-381.

[3] K. I. Babenko, On a local existence theorem in the theory of surface waves of finite amplitude, Soviet Math. Doklady 35 (1987), 647-650.

[4] Some remarks on the theory of surface waves of finite amplitude, Soviet Math. Doklady 35 (1987), 599-603.

[5] K. I. Babenko, V. Yu. Petrovich, and A.I. Rakhmanov, A computational experiment in the theory of surface waves of finite amplitude, Soviet Math. Dokl. 38 (1989), $327-331$.

[6] _ On a demonstrative experiment in the theory of surface waves of finite amplitude, Soviet Math. Dokl. 38 (1989), 626-630.

[7] C. Baesens and R. S. MacKay, Uniformly travelling water waves from a dynamical systems viewpoint : some insight into bifurcations from Stokes' family, J. Fluid Mech. 241 (1992), 333-347.

[8] B. Buffoni, E. N. Dancer, and J. F. Toland, The regularity and local bifurcation of steady periodic water waves, Arch. Rational Mech. Anal. 152 (2000), 207-240.

[9] _ The sub-harmonic bifurcation of Stokes waves, Arch. Rational Mech. Anal. 152 (2000), 241-271.

[10] B. Buffoni, É. Séré, and J. F. Toland, Minimization methods for quasi-linear problems, with an application to periodic water waves, in preparation.

[11] B. Buffoni and J. F. Toland, Dual free boundaries for Stokes waves, Comptes Rendus Acad. Sci. Paris, Série 1332 (2001), 73-78.

[12] B. Chen and P. G. Saffman, Numerical evidence for the existence of new types of gravity waves of permanent form on deep water, Studies in Appl. Math. 62 (1980), $1-21$.

[13] W. Craig and P. Sternberg, Symmetry of solitary waves, Comm. Partial Differential Equations 13 (5) (1988), 603-633.

[14] I. Ekeland and N. Ghoussoub, Selected new aspects of the calculus of variations in the large, Bull. Amer. Math. Soc. (N.S.) 39(2) (2002), 207-265.

[15] P. R. Garabedian, Surface waves of finite depth, J. d'Anal. Math. 14 (1965), 161-169.

[16] N. Ghoussoub, Duality and Perturbation Methods in Critical Point Theory, Cambridge University Press, Cambridge, 1993. 
[17] H. Hofer, A geometric description of the neighborhood of a critical point given by the mountain pass theorem, J. London Math. Soc. 31 (1985), 566-570.

[18] M. S. Longuet-Higgins, Bifurcation in gravity waves, J. Fluid Mech. 151 (1985), $457-475$.

[19] P. I. Plotnikov, Justification of the Stokes hypothesis in the theory of surface waves, Soviet Phys. Dokl. 28 (3) (1983), 232-333.

[20] M. Schechter, The Hampwile theorem for nonlinear eigenvalues, Duke Math. 59 (1989), 325-335.

[21] E. R. Spielvogel, A variational principle for waves of infinite depth, Arch. Rational Mech. Anal. 39 (1970), 189-205.

[22] J. F. Toland, Continuity and differentiability of Nemytskii operators on the Hardy space $H^{1,1}\left(T^{1}\right)$, To appear in Arkiv für Mathematik.

[23]_ On a pseudo-differential equation for Stokes waves, Preprint, 2001.

[24] _ Stokes waves in Hardy spaces and as distributions, J. Math. Pure et Appl. 79(9) (2000), 901-917.

[25] R. E. L. Turner, A variational approach to surface solitary waves, Journal of Differential Equations 55 (1984), 401-438.

[26] A. Zygmund, Trigonometric Series I 6 II, Cambridge University Press, Cambridge, 1959. 